\title{
Diagnóstico de los costos de no calidad en el área de Regiduría de Pisos del hotel Iberostar Grand Packard
}

DOI: https://doi.org/10.33262/ap.v3i1.38

\begin{abstract}
(c) (1) (8)(2)
Diagnosis non-quality costs in the area of flooring at the Packard Iberostar Grand hotel
\end{abstract}

Dolores Anay Matos Murcia ${ }^{1}$

\begin{abstract}
.
This research was carried out at the Packard Iberostar Grand hotel of category 5 stars belonging to the Gaviota S.A. Tourism Group and administered by the extractive chain Iberostar, with the aim of diagnosing non-quality costs in the area of flooring. In order to meet the objective set, theoretical and empirical methods of research were used, within the latter, the documentary analysis to characterize the hotel under study, the interview with the quality specialist to find out about the work that is carried out in the installation in relation to the quality and costs associated with it, the feedback mechanisms that are used to determine the satisfaction of the clients.The head of housekeeper was also interviewed to learn about the operation of the flooring area, as well as a survey of a sample of waitresses to learn about the internal clients' impact on quality of service. The results were processed using parametric statistics which identified a set of nonconformities that cause non-quality internal and external failure costs. The intangible cost of clients' dissatisfaction in 2019 could then be accounted for. Finally 13 improvement actions were proposed.
\end{abstract}

Keywords: Quality, cost, clients, flooring area.

\section{Resumen.}

\footnotetext{
${ }^{1}$ Universidad de La Habana, Facultad de Turismo. La Habana, Cuba.rolivera@ftur.uh.cu
} 
La presente investigación se llevó a cabo en el hotel Iberostar Grand Packard de categoría 5 estrellas perteneciente al Grupo de turismo Gaviota S.A y administrado por la cadena extrajera Iberostar, con el objetivo de diagnosticar los costos de no calidad en el área de Regiduría de Pisos. Con el fin de cumplir el objetivo trazado se emplearon métodos teóricos y empíricos de investigación, dentro de estos últimos, el análisis documental para caracterizar el hotel objeto de estudio, la entrevista a la especialista de calidad para indagar sobre los trabajos que se realizan en la instalación con relación a la calidad y los costos asociados a esta, los mecanismos de retroalimentación que se utilizan para determinar la satisfacción de los clientes, además de sus quejas y reclamaciones. También se entrevistó al Ama de Llaves para conocer sobre el funcionamiento del área de Regiduría de Pisos y se aplicó una encuesta a una muestra de camareras para conocer la incidencia del cliente interno en la calidad del servicio. Los resultados se procesaron mediante la estadística paramétrica los cuales permitieron identificar un conjunto de no conformidades que originan costos de no calidad de falla interna y externa. Fue posible además contabilizar el costo intangible que se produjo por la insatisfacción de clientes en el año 2019. Luego a partir de todos los resultados se propusieron 13 acciones de mejoras.

Palabras claves: Calidad, costo, clientes, regiduría de pisos.

\section{Introducción.}

El entorno globalizado y competitivo exige a las empresas un esfuerzo constante en mejorar sus procesos, de tal manera que muchas de ellas están implementando programas de mejora o calidad total en su organización. Esto sin excluir las acciones de reingeniería, para reestructurar y lograr la innovación que les permita: mantenerse actualizados en la satisfacción a los requerimientos de sus clientes, ser competitivos y flexibles a los cambios; ser veloz más que grande, pues esto significará la diferencia entre subsistir o desaparecer (Flores y Humberto, 2015).

Ante esta situación, las empresas que quieran sobrevivir han de ser competitivas, y para esto tienen que ser líderes en innovación, tecnología, calidad y costos. Por tanto, se hace necesario asegurar el crecimiento y sostenibilidad a largo plazo en el mercado, visualizando la gestión de la calidad como herramienta necesaria e imprescindible y asociada de manera directa a los costos, los cuales tienen que ser identificados y medidos para poder justificar las inversiones que se deben desarrollar para reducirlos (Casermeiro, Scheuber y Varas, 2010).

En este sentido Mahal y Hossain (2015) afirman que poseer elementos tan fuertes como son los costos asociados a la función de la calidad dentro de una organización posibilita el trazado de estrategias de mejoras con la consiguiente elevación de los índices de rentabilidad, eficiencia y eficacia. Estos autores también exponen que por lo general muchas de las empresas con sistemas de calidad certificados no poseen un sistema de costos de la calidad, limitándose a clasificarlos solamente en sus manuales sin prestarles la debida atención. 
Los altos mandos, la gerencia y el departamento administrativo se enfrentan constantemente con diferentes situaciones que afectan el funcionamiento de la empresa. La información que se obtenga acerca de los costos y los gastos en que incurre la organización para realizar su actividad y que rigen su comportamiento son de vital importancia para la toma de decisiones de manera rápida y eficaz. Esto hace que en la actualidad la contabilidad viva una nueva época marcada por la demanda de más cantidad de información (Yera, Tellería, Hernández y col., 2015).

El sector turístico no escapa a la realidad de la globalización, siendo necesario manejar información relevante y oportuna en materia de costos, como pieza clave para desarrollar ventajas, sin embargo, el tema de los costos de calidad en Cuba no está enraizado en la cultura empresarial y ha sido poco tratado. Los sistemas contables de las organizaciones cubanas no están diseñados para identificar los costos de calidad y esta es una de las razones por las cuales la alta gerencia es mucho más sensible a los costos globales de la producción que a los de calidad.

El Grupo de Turismo Gaviota SA. inmerso en esta realidad ha incluido dentro de su banco de problemas para el desarrollo de investigaciones el estudio de los costos asociados a la calidad, con énfasis en sus hoteles de lujo, siendo el hotel Iberostar Grand Packard, de categoría 5 estrellas una de sus instalaciones donde existen no conformidades que afectan la calidad del servicio, en particular en el área de Regiduría de Pisos, pero no están identificados los costos de no calidad que se producen a partir de estas.

Los cuestionamientos serían ¿Cuáles son los factores que originan costos de no calidad en el área de regiduría de pisos? ¿Qué costos son internos o externos? ¿Cómo disminuirlos?

Se define, así como objetivo del presente documento: Diagnosticar los costos de no calidad en el área de Regiduría de Pisos del hotel Iberostar Grand Packard.

El cumplimento de este objetivo, a partir de la identificación de los factores que originan costos de no calidad en el área de Regiduría de Pisos del hotel Iberostar Grand Packard, permitirá estructurar el marco lógico para la mejora, así como proponer acciones de mejoras en función de la planificación y el control de esta área dentro de la instalación.

\section{Metodología.}

La estrategia metodológica diseñada y seguida durante el proceso investigativo, se basó en la investigación-acción, cuyo propósito fundamental según Hernández, Fernández y Baptista (2010) se centra en aportar información que guíe la toma de decisiones para programas, procesos y reformas estructurales. En este sentido fueron aplicados los siguientes métodos:

\section{De orden teórico:}


Histórico lógico: Permitió conocer los costos asociados a la calidad y su clasificación, los aspectos generales del proceso de alojamiento dentro de la actividad turística y en particular del área de Regiduría de Pisos, así como la importancia de la medición de los costos de no calidad en dicha área.

Análisis y síntesis: A partir de la revisión de la literatura y documentación especializada se pudo conformar las bases teóricas conceptuales para el mejor entendimiento y caracterización del fenómeno estudiado.

Hipotético - deductivo: Posibilitó como resultado de la investigación previa sobre el tema, formular la hipótesis de la investigación, y luego, a través de inferencias lógicas deductivas, derivar que la determinación de los factores que originan costos de no calidad en el área de Regiduría de Pisos permite proponer acciones de mejoras.

\section{De orden empírico:}

Análisis documental: Se revisó la documentación de la Cadena Iberostar y del Grupo Gaviota, en específico el Folleto de servicios de Iberostar y el Manual de acogida de Gaviota. Del hotel se consultó el Modelo de caracterización y el Manual de procedimientos de pisos, Versión 1 (2019) y la Resolución 342/2019, el Estado de Resultado del año 2019 y la Bitácora de Relaciones Públicas.

Entrevista (semiestructurada): Se entrevistó a la especialista de calidad de la instalación para conocer la gestión de la calidad y a la jefa de Ama de Llaves para indagar sobre los procesos en el área de Regiduría de Pisos.

Encuesta: Se utilizó la encuesta de satisfacción de cliente interno diseñada por el corporativo de Iberostar, para identificar los factores negativos que impiden un correcto funcionamiento del área de Regiduría. Fue aplicada a una muestra de 19 camareras de ellas 13 de habitaciones y 6 de áreas comunes. La cantidad de encuestados se determinó a partir del programa Sample Size Calculator, considerando como población la cantidad de trabajadores totales del área de Regiduría de Pisos (54), un nivel de confianza del 90\% y un error máximo de estimación del $10 \%$.

\section{Métodos estadísticos:}

\section{Estadística paramétrica:}

Los resultados se procesaron a través del cálculo de frecuencia y porcentaje.

\section{Clasificación de los costos asociados a la calidad.}

Se entiende por costo el valor de los recursos económicos utilizados para la producción de un determinado producto o la prestación de un servicio, pero cuando se asocia a la calidad se conceptualiza como lo que le cuesta a una organización desarrollar la función de la calidad 
Fernández y Escalona (2014). En este sentido Colunga y Saldierna (1994), Asociación Española de Contabilidad y Administración (AECA, 1995), Sarmiento (2010), Casermeiro, Scheuber y Varas (2010), Cuatrecasas, Carrión, Carvajal y col., (2010), Thomasson y Wallin (2013), Flores y Humberto (2015), Hernández, Tellería, Yera y col., (2015), Shrouty y Tiwari (2017), American Society for Quality (ASQ, 2018), Sower y Broussard (2018) dividen los costos en dos grupos: de calidad y no calidad.

1. Costos de calidad: Costos incurridos en el diseño, implementación, operación y mantenimiento de los sistemas de calidad de una organización, aquellos costos de la organización comprometidos en los procesos de mejoramiento continuo, y los costos del sistema, productos y servicios fracasados porque no han tenido el éxito esperado en el mercado. Estos se clasifican en costos de prevención y evaluación:

2. Costos de prevención: Los costos de prevención son los gastos realizados para evitar que se cometan errores. Son una inversión a futuro, inversión para evitar costos de la no calidad.

3. Costos de evaluación: Cantidad que se gasta en inspección y control. Dígase: inspecciones, pruebas y otras evaluaciones planificadas.

4. Costos de no calidad: Denominados también de no conformidad. Los agrupan en costos de fallas externas e internas. Estos a su vez no son controlables por la empresa. Su explicación se muestra a continuación:

5. Costos de fallas internas: Se definen como el costo en que incurre la empresa como consecuencia de los errores detectados antes que el producto/servicio sea entregado al cliente. Por tanto, son los costos que se producen en el producto/servicio antes de salir de la organización.

6. Costos de fallas externas: Son en los que incurre la empresa cuando se detecta algún problema en los productos/servicios posteriores a la venta; o sea una vez que es entregado al cliente. Se trata de los costos asociados porque el producto o servicio no satisface al cliente o no cumplen con los requisitos de calidad.

Sin embargo, Pyzdek (1996), Campanella (1997) y Crosby (1997) estiman que todos los costos son de calidad y dentro de estos se encuentran tanto los de prevención y evaluación como los de fallas o fracasos clasificados igualmente en fallas internas y externas.

La AECA (1995), Meneses y Rodríguez (2010) consideran los siguientes elementos componentes de los costos de fallas.

Elementos que componen los costos por fallas internas: Mermas y pérdidas de insumos, gastos de reprogramación, gastos de reinspección, tiempo de gestionar fallos en las certificaciones y resolución de contratos, reprocesos, pérdidas de materiales incontrolados, desperdicios, reparaciones, exceso de consumos de energía y materiales, errores en la selección de personal, desmotivación de la plantilla.

Elementos que componen los costos por fallas externas: Devoluciones, pérdida de ventas, reclamaciones de clientes, indemnizaciones, penalizaciones, tratamiento de quejas de los 
clientes, pérdida de ventas por mala imagen, costos de responsabilidad, multas por incumplimiento de legislaciones y contratos.

Tal y como se ha visto en la clasificación anterior existen muchos componentes que constituyen costos para cualquier empresa que incurra en ellos. Con respecto a estos componentes Casermeiro, Scheuber y Varas (2010) consideran que los principales problemas existentes en su contabilización, planificación y control es la falta de criterios convencionales para calcularlos. Debido a este problema resulta necesario clasificar estos de la siguiente forma:

- Costos tangibles: Son aquellos que se pueden calcular con criterios convencionales de costos, normalmente siguiendo principios de contabilidad.

- Costos intangibles: También denominados costos implícitos, son aquellos que se calculan con criterios subjetivos y que no son registrados como costos en los sistemas de contabilidad.

Aceptar la existencia de los costos de no calidad como parte integral de los costos en los que incurre cualquier empresa es solo un primer paso. Su verdadero valor radica en identificarlos, investigar los factores que los generan a fin actuar en función de disminuirlos

Importancia de la medición de los costos de no calidad en el Área de Regiduría de pisos.

El análisis de los costos de no calidad proporciona ventajas tanto para las empresas industriales como de servicio, por cuanto permite contabilizarlos y tomar medidas para disminuirlos. De acuerdo con Gallego (2007) permite un mejor aprovechamiento de los recursos humanos y materiales y por consiguiente eleva el rendimiento de las empresas. Agrega que específicamente en el área de Regiduría de Pisos el control de los costos de no calidad juega un rol fundamental porque contribuye de manera directa a las utilidades del proceso de alojamiento y por ende de la instalación.

Planteamiento que confirma Del Toro (2011) quien expone que en la actualidad la relevancia alcanzada por el puesto de Ama de Llaves, ha permitido que la capacitación y la planificación, sean pilares básicos para el cumplimiento de los requerimientos que se establecen para el área de Regiduría de Piso, logrando que el trabajo se realice con eficiencia y no se originen costos de no calidad, lo que de producirse disminuyen los beneficios de la instalación, pudiendo afectar incluso la imagen de la misma.

Según Lattin (2012) la información del funcionamiento de un hotel y de los costos de cada servicio es fundamental para la toma de decisiones acertadas, por tanto, se debe hacer énfasis en una correcta gestión de costos. De hecho, Morillo y Marysela (2010), González Arias, Frías-Jiménez y Gómez-Figueroa (2014), Prado-Chaviano, Cruz-Suárez, Baujín-Pérez y col., (2016) han desarrollado procedimientos para el cálculo de los costos de no calidad en hoteles y le otorgan un valor primordial al proceso de alojamiento y dentro de este al área de Regiduría de Pisos como subproceso que mayores quejas genera. 
Otros autores como Ulacia (2016) y Domínguez (2019) afirman que la falta de rigor, la rutina diaria, la insuficiente utilización de los métodos y herramientas, que norman y evalúan los procedimientos del área de Regiduría de Pisos, son algunos de los factores que afectan la calidad en las instalaciones turísticas, originan quejas y reclamaciones en los huéspedes y por consiguiente impiden el alcance del máximo beneficio; siendo ahí donde cobra importancia el nivel de exigencia de los directivos.

Estas últimas dos autoras plantean, además, que uno de los aspectos a tener en cuenta es que la operación del área de Regiduría de Pisos es diaria y si una habitación no se vende se generan gastos imposibles de evitar, los que impactan de forma desfavorable, en los ingresos del establecimiento, de ahí la necesidad de una buena gestión de habitaciones, lo que se logra ofreciendo un servicio sin fallas que ocasionen costos de no calidad.

Haciendo coincidir la importancia de los costos de no calidad y el papel del área de Regiduría de Pisos, no cabe dudas que identificar estos y descubrir los factores que lo generan es una base a la toma de decisiones y la mejora de la gestión en esta área.

\section{Resultados.}

Valoración de los costos de no calidad. Resultados de la entrevista realizada a la especialista de calidad y a la jefa de Ama de Llaves

Como resultado de la entrevista a la especialista de calidad se pudo conocer que en la instalación no se determinan los costos de calidad y la mayoría del personal no tiene claridad en relación al tema. Aunque si se conoce que cuando se incumplen los requisitos de calidad en cualquiera de los procesos del hotel origina un costo que atenta contra la rentabilidad de la instalación. Agrega que es importante determinarlos porque ello permite tomar acciones de mejora más efectivas.

La especialista de además manifestó que el sistema de retroalimentación de la instalación no incluye el tradicional libro de quejas y sugerencias, encuestas de satisfacción ni entrevistas a los clientes externos, dado que la cadena Iberostar considera que esto ocasiona molestia y gasto de tiempo a los huéspedes, y por tanto no lo permite en hoteles que sustentan la categoría GRAND. La retroalimentación se realiza a través de la asistencia vía telefónica, la página oficial del hotel en las redes sociales, los sitios web como Tripadvisor y el análisis de los resultados del Review Pro.

\section{Ausentismo:}

Se consultó las prenóminas de pago del año 2019, donde se registra el resumen de las ausencias de todo el personal, afectándose el hotel por el número de ausencias injustificadas y justificadas, estas últimas, atendiendo al gran número de certificados médicos presentados. En la figura 1 se visualiza el comportamiento de las ausencias en el área. 
Figura 1. Comportamiento de las ausencias en el área de Regiduría de Pisos.

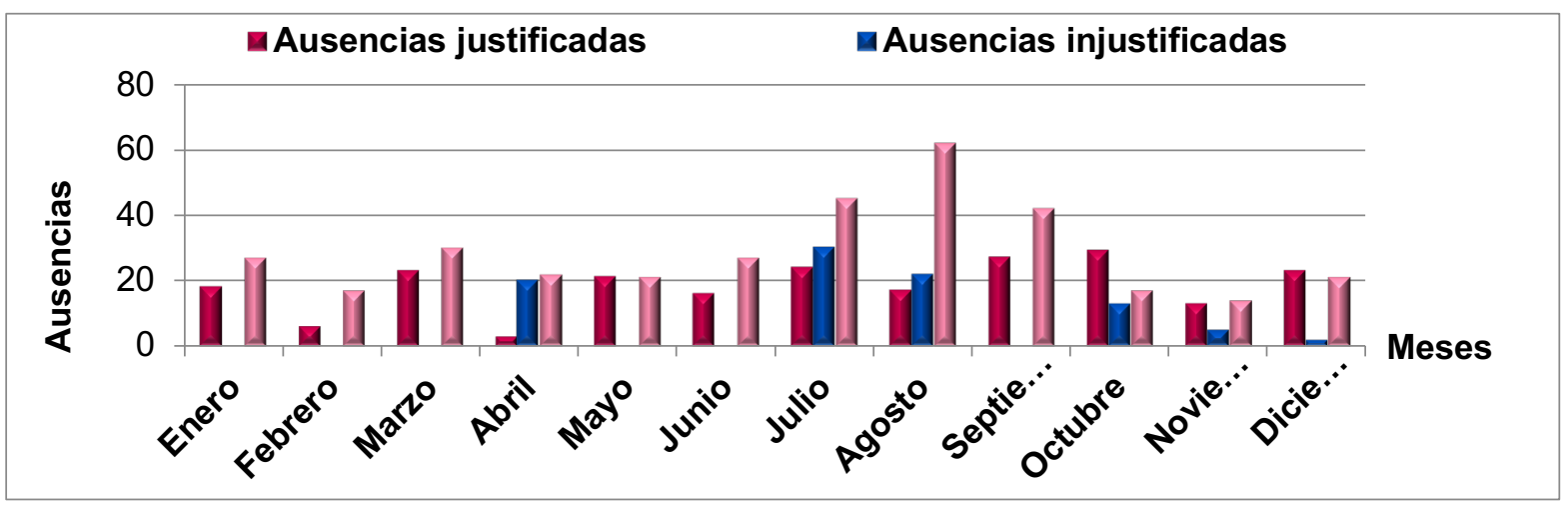

Fuente: Prenóminas de pago del Hotel Iberostar Grand Packard (2019).

Los certificados médicos aun cuando son ausencias justificadas se graficaron por separados para hacer más visible la incidencia que estos poseen. Los meses con mayor cantidad de ausencias por certificados médicos fueron julio con 45 y agosto con 62. Este valor es superior a los 31 días que poseen ambos meses teniendo en cuenta que puede estar ausente en un mismo período de tiempo más de una camarera. Las ausencias injustificadas afectan en menor medida, siendo julio y agosto también los meses de mayor cantidad de ausencias injustificadas con 30 y 22 respectivamente.

\section{Reproceso en el servicio.}

El reproceso ocurre después de la supervisión de las habitaciones y áreas donde se detectan errores, también cuando el personal de mantenimiento realiza su trabajo y no tiene cuidado al hacerlo. La entrevista realizada al Ama de Llaves permitió conocer la existencia de reprocesos ocasionados por tener que realizar nuevamente el servicio, ya sea la limpieza de habitaciones, pasillos o escaleras porque los resultados no estuvieron de acuerdo con lo establecido.

\section{Ineficiente capacitación a los trabajadores.}

De acuerdo al Estado de Resultado de la instalación correspondiente al año 2019 se designó como presupuesto para los gastos de capacitación y adiestramiento un total de $\$ 30004.5$ MLC, del cual solo se consumieron \$3 671.415 MLC; para un cumplimiento de $12.24 \%$ con respecto al plan. Estos resultados muestran una diferencia considerable entre el plan y lo real que, si bien en materia de costo global es un "ahorro", esto se pudiera analizar por cuanto implica que no se están ofreciendo todas las acciones de capacitación programadas, las que se concibieron a partir de la necesidad de elevar los conocimientos del personal, para que pudieran desempeñar mejor sus funciones y ofrecer un servicio acorde con la categoría del hotel.

Gasto de energía eléctrica. 
El hotel tiene un sistema de control automático, a partir del cual se controla todos los portadores energéticos del mismo, dígase: agua, gas y electricidad. Dicho sistema reporta el comportamiento de estos portadores energéticos de manera general por lo que no se puede identificar el comportamiento de estos por cada departamento. En el caso específico de las habitaciones, como parte de la tecnología que requiere un hotel de lujo, existe un sensor automático a través del cual la habitación una vez que está ocupada se enciende y por el contrario se apaga. Por esta razón dentro de los pasos establecidos para la limpieza de habitación está concebido apagar todos los dispositivos con que esta cuenta. Sin embargo, según manifestó la Ama de Llaves en reiteradas ocasiones las camareras para realizar la limpieza de las habitaciones no apagan los aires, ni las luminarias, encienden el televisor o demás dispositivos para escuchar música. Esto constituye una violación precisamente por aumentar el consumo de electricidad de la instalación.

\section{Resultados de la encuesta.}

También fue aplicada una encuesta de Clima Laboral para conocer aspectos relacionados con la satisfacción de los trabajadores. La caracterización de dicha muestra se presenta a continuación (Tabla 1). Como se aprecia el 100\% es del sexo femenino, la mayoría con una edad comprendida en el rango de 21 a 40 años. La totalidad de los encuestados tienen un nivel escolar apropiado y formación especializada obtenida en las diferentes escuelas del sistema Formatur del Mintur y la mayoría poseen entre 1 y 10 años de experiencia en la labor que realizan.

Tabla 1. Caracterización de la muestra encuestada.

\begin{tabular}{|c|c|c|}
\hline Variables & Categorias de respuesta & $\%$ \\
\hline \multirow{2}{*}{ Sexo } & Femenino & 100 \\
\hline & Masculino & 0 \\
\hline \multirow{3}{*}{ Edad } & 21 a 40 & 89.47 \\
\hline & 41 a 60 & 10.53 \\
\hline & Más de 60 & 0 \\
\hline \multirow{2}{*}{ Nivel escolar } & 12mo Grado & 57.89 \\
\hline & Técnico medio & 42.11 \\
\hline Formación especializada & Sí & 100 \\
\hline \multirow{2}{*}{ Años de experiencia } & 1 a 10 & 73.68 \\
\hline & 15 a 20 & 26.32 \\
\hline
\end{tabular}

Fuente: Elaboración propia.

En cuanto a las posibilidades reales de promoción que se tienen dentro del hotel la figura1 muestra que el $15.8 \%$ de los encuestados consideran que sí existen posibilidades reales de promoción, el $31.6 \%$ cree que sí, un 47,4\% estima que no mientras que el 5.3\% afirma que no existen posibilidades reales de promoción dentro del hotel. Se aprecia que para la mayoría la instalación no es un lugar que favorezca su promoción. 
Figura 1. Respuesta de los encuestados sobre las posibilidades de promoción.

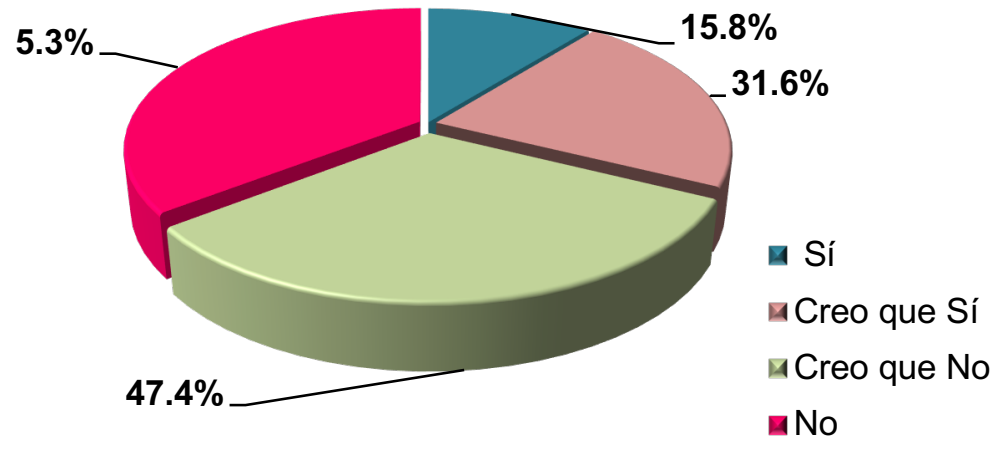

Fuente: Elaboración propia.

De igual manera las respuestas obtenidas en relación a las oportunidades que se le brindan para su superación fueron heterogéneas en cuanto al conocimiento de las acciones que se planifican y el efecto positivo que puedan tener estas en su desempeño profesional. La figura 2 exhibe los resultados del conocimiento que tienen los encuestados de los programas de entrenamiento del hotel. Se observa que solo el 36,9\% plantea conocerlos, el 15, $8 \%$ tiene duda al respecto y el 47,3\% no los conoce.

Figura 2. Conocimiento de los encuestados de los programas de entrenamiento.

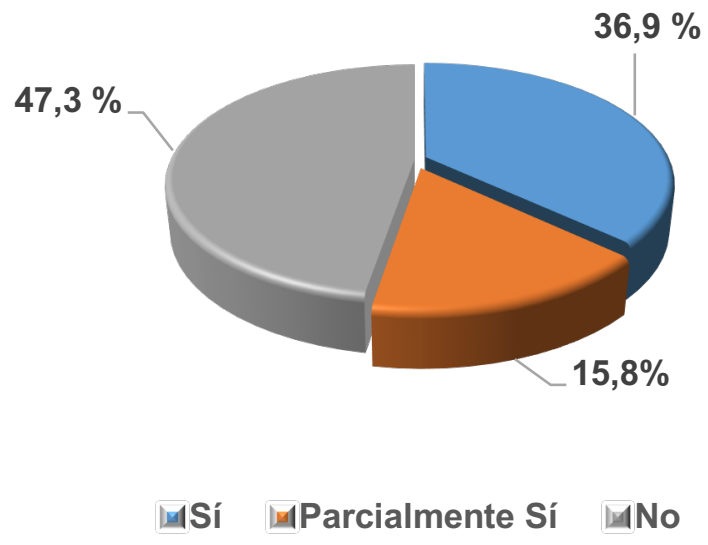

Fuente: Elaboración propia.

La figura 3 muestra que el $26.4 \%$ de los trabajadores encuestados estiman que el entrenamiento que reciben sí contribuye de manera positiva a la mejora de su trabajo, mientras que el $31,7 \%$ estima que no y el $36.9 \%$ opina que no todas las acciones que se realizan favorecen su desempeño laboral. Se apreció un interés marcado de los trabajadores por su superación, el $100 \%$ tiene deseos y disposición para ello y están conscientes que en la medida que estén más preparados, brindarán un mejor servicio al cliente. 
Figura 3. Respuesta de los encuestados sobre el erecto de su entrenamiento laboral.

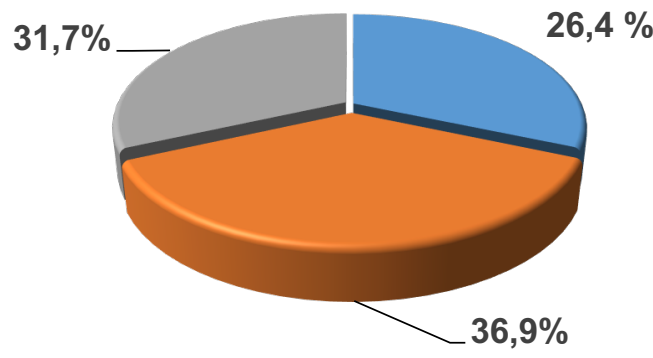

四 Sí linParcialmente Sí 四No

Fuente: Elaboración propia.

Los resultados anteriores evidencian la necesidad de brindar oportunidades de formación que sirvan a los trabajadores para mejorar su posición dentro de la organización y de lograr una mayor efectividad en las actividades de capacitación que se conciben en el hotel, dado la incidencia que tienen estos elementos en la ocurrencia de costos de no calidad de fallas internas por desconocimiento y falta de destreza del personal al realizar sus funciones.

\section{Análisis de las quejas de clientes externos}

Tabla 2. Quejas recibidas de los clientes en el año 2019.

\begin{tabular}{|c|c|c|c|c|c|c|}
\hline \multirow[b]{2}{*}{ Meses } & \multicolumn{5}{|c|}{ Categorias } & \multirow{2}{*}{$\begin{array}{c}\text { Total de } \\
\text { quejas }\end{array}$} \\
\hline & Limpieza & Decoración & $\begin{array}{c}\text { Reposición de } \\
\text { amenidades }\end{array}$ & Lavandería & $\begin{array}{l}\text { Avituallamiento } \\
\text { de habitación }\end{array}$ & \\
\hline Enero & 5 & & 1 & 1 & & 7 \\
\hline Febrero & 3 & & & & & 3 \\
\hline Marzo & & & & 2 & & 2 \\
\hline Abril & 4 & & & 2 & & 6 \\
\hline Mayo & 1 & & & 3 & & 4 \\
\hline Junio & 5 & & & 2 & 1 & 8 \\
\hline Julio & 9 & 2 & 1 & 7 & 8 & 27 \\
\hline Agosto & 16 & 1 & 3 & 4 & 9 & 33 \\
\hline Septiembre & 2 & & & & 2 & 4 \\
\hline Octubre & 2 & & & & 4 & 6 \\
\hline Noviembre & 2 & & & & 1 & 3 \\
\hline Diciembre & 3 & & & & 2 & 5 \\
\hline Total & 52 & 3 & 5 & 21 & 27 & 108 \\
\hline$\%$ & 48.15 & 2.78 & 4.63 & 19.44 & 25 & \\
\hline
\end{tabular}

Fuente: Bitácora de Relaciones Públicas de hotel Iberostar Grand Packard.

Se aprecia que es la categoría limpieza la de mayor incidencia con un total de 52 quejas lo que representa el $48.15 \%$ del total, valor que excede considerablemente al obtenido para el resto de las categorías. Al analizar las quejas más frecuentes (limpieza insuficiente de la 
habitación, el baño y las salas nobles) se advierte un mal trabajo de las camareras, falta de exigencia de los directivos e incumplimiento de los requisitos establecidos en las normativas higiénicas sanitarias, las cuales son de obligatorio cumplimiento por la repercusión que tienen en la calidad percibida por el cliente.

Es preocupante también las quejas en relación al avituallamiento de habitaciones y la lavandería, con $25 \%$ (27 incidencias) y $19.44 \%$ (21 incidencia) respectivamente, por cuanto se trata de un hotel cinco estrellas de lujo, comercializado bajo la marca GRAND, con un precio elevado justamente, por lo que las habitaciones tienen que contar con todo lo que requiere la categoría que ostenta el hotel y velar por la calidad de todos por los servicios que ofrece. Luego se tiene que garantizar el abastecimiento de los servicios.

Aunque las incidencias atribuidas a la decoración de las habitaciones y la reposición de amenidades fueron pocas, también hay que prestarle atención, por cuanto hoy el cliente emite sus criterios a través de las redes sociales siendo éstas ampliamente consultadas a nivel internacional, y el juicio de un cliente insatisfecho puede influir en un número considerables de clientes potenciales.

Para atenuar las molestias ocasionadas a los huéspedes la instalación realizó un total de 108 compensaciones con un costo total de no calidad de \$ 2129.000 MLC, siendo los meses más sobresalientes julio y agosto, con valores de \$310.24 MLC y 769.50 MLC respectivamente (Tabla 3).

Tabla 3. Compensaciones realizadas en el año 2019

\begin{tabular}{|c|c|c|c|c|}
\hline \multirow[t]{2}{*}{ Meses } & \multirow[t]{2}{*}{ Tipo de Compensación } & \multirow[t]{2}{*}{ Cantidad } & \multicolumn{2}{|c|}{ Costo (MLC) } \\
\hline & & & Unitario & Total \\
\hline \multirow[t]{3}{*}{ Enero } & Late check-out & 5 & 45.00 & 225.00 \\
\hline & Havana Club 3 años & 2 & 5.88 & 11.76 \\
\hline & Platos de frutas & & & \\
\hline Febrero & Havana Club 3 años & 3 & 5.88 & 17.64 \\
\hline & Platos de frutas & & & \\
\hline Marzo & Havana Club 3 años & 2 & 5.88 & 11.76 \\
\hline & Platos de frutas & & & \\
\hline \multirow[t]{4}{*}{ Abril } & Late check out & 2 & 45.00 & 90.00 \\
\hline & Havana Club 3 años & 1 & 5.88 & 5.88 \\
\hline & Platos de frutas & & & \\
\hline & Masaje de 60 minutos & 3 & 50.00 & 150.00 \\
\hline Mayo & Havana Club 3 años & 4 & 5.88 & 23.52 \\
\hline & Chocolates & & & \\
\hline Junio & Havana Club 3 años & 5 & 5.88 & 29.40 \\
\hline
\end{tabular}




\begin{tabular}{|c|c|c|c|c|}
\hline & Chocolates & & & \\
\hline & Masaje (60 minutos) & 2 & 50.00 & 100.00 \\
\hline & Upgrade de habitación & 1 & 80.00 & 80.00 \\
\hline \multirow[t]{3}{*}{ Julio } & Havana Club 3 años & 23 & 5.88 & 135.24 \\
\hline & $\begin{array}{l}\text { Chocolates } \\
\text { Upgrade de habitación }\end{array}$ & 1 & 40.00 & 40.00 \\
\hline & Late check-out free & 3 & 45.00 & 135.00 \\
\hline \multirow[t]{4}{*}{ Agosto } & $\begin{array}{l}\text { Havana Club } 3 \text { años } \\
\text { Chocolates }\end{array}$ & 19 & 5.88 & 111.72 \\
\hline & $\begin{array}{lrrr}\text { Havana } & \text { Club } & 3 & \text { años } \\
\text { Botella } & \text { de } & & \text { agua } \\
\text { Chocolates } & & & \end{array}$ & 1 & 8.88 & 8.88 \\
\hline & $\begin{array}{l}\text { Cena en el Restaurante } \\
\text { Biscuit }\end{array}$ & 1 & 108.90 & 108.90 \\
\hline & Late check out & 12 & 45.00 & 540.00 \\
\hline \multirow[t]{2}{*}{ Septiembre } & Late check-out free & 2 & 45.00 & 90.00 \\
\hline & $\begin{array}{l}\text { Havana Club } 3 \text { años y } \\
\text { chocolates. }\end{array}$ & 2 & 5.88 & 11.76 \\
\hline \multirow[t]{3}{*}{ Octubre } & Almuerzo ( Snack- Bar) & 1 & 128.70 & 128.70 \\
\hline & $\begin{array}{l}\text { Botella de vino tinto } \\
\text { Frontera }\end{array}$ & 1 & 3.28 & 3.28 \\
\hline & $\begin{array}{l}\text { Havana Club } 3 \text { años } \\
\text { Chocolates. }\end{array}$ & 4 & 5.88 & 23.52 \\
\hline Noviembre & $\begin{array}{l}\text { Havana Club } 3 \text { años } \\
\text { Chocolates }\end{array}$ & 3 & 5.88 & 17.64 \\
\hline Diciembre & $\begin{array}{l}\text { Havana Club } 3 \text { años } \\
\text { Chocolates }\end{array}$ & 5 & 5.88 & 29.40 \\
\hline Total & --------------- & 108 & & $\$ 2129.00$ \\
\hline
\end{tabular}

Fuente: Bitácora de relaciones Públicas del hotel Iberostar Grand Packard

Considerando que los clientes que emitieron quejas están insatisfechos con el servicio del hotel y no van a regresar a este, se estimó el costo de no calidad por clientes perdidos, que se clasifica como un costo intangible de falla externa. Para ello se empleó la fórmula siguiente:

Costo intangible $=(\mathrm{PM})(\mathrm{EM})(\mathrm{CP})$

Siendo:

PM: Precio medio de habitación $=\$ 315$ MLC 
EM: Estancia media en el hotel $=2.5$

CP: Número de clientes perdidos $=108$

Costo intangible $=(\$ 315)(2.5)(108)$

Costo intangible $=\$ 85050.00 \mathrm{MLC}$

El hotel ha perdido un total de $\$ 85050.00 \mathrm{MLC}$, lo que no solo es alarmante por lo que representa desde el punto de vista económico para el hotel, sino por lo que significa para el Grupo Hotelero en cuanto a pérdida de imagen, la cual hoy es más susceptible que nunca como se ha mencionado debido al nivel de información que brindan las redes sociales.

\section{Propuesta de acciones de mejoras:}

1. Impulsar el trabajo por la calidad en el hotel a fin de implementar el Sistema de Gestión de la calidad en un período corto de tiempo.

2. Sensibilizar al departamento de Economía de la necesidad de identificar y contabilizar los costos de no calidad y regístralos en una partida independiente a la de Atención a Clientes.

3. Confeccionar un procedimiento que permita la detección de los costos de no calidad en el área de Regiduría de Pisos, y brinde además las herramientas para la cuantificación de los mismos.

4. Concebir una cuenta denominada Clientes Perdidos expresada en físico y Clientes Perdidos expresada en valor, que permita abrir centros de costos una vez que se identifiquen las no conformidades que lo originan.

5. Establecer alianzas con la Escuela Ramal y el Centro de Capacitación del Mintur, a fin de conciliar el aprendizaje continuo de los trabajadores en temas a fines a su desempeño profesional.

6. Capacitar al personal del área de Regiduría de Pisos sobre los costos de no calidad y su implicación para el hotel.

7. Mejorar los procesos de reclutamiento, selección y formación de los empleados evaluando las diferencias individuales y variables contextuales que se relacionan con una mejor calidad del servicio.

8. Establecer relaciones entre las exigencias físicas elevadas del puesto de trabajo y el ausentismo por lesiones y, entre el ausentismo de corta duración y su justificación médica. 
9. Mantener una vigilancia estricta y elevar la exigencia sobre las diferentes actividades que realiza el personal del área de Regiduría de Pisos, con énfasis en los aspectos relacionados con la limpieza.

10.Incitar el trabajo colectivo, haciendo entender a todos los trabajadores que sus acciones no son aisladas, sino parte de un sistema que engloba a toda la organización.

11.Fomentar la comunicación interna y el Team building.

12. Sensibilizar a los directivos del hotel de la necesidad de establecer mecanismos efectivos para estimular y reconocer a los mejores trabajadores.

13. Exigir el cumplimiento estricto de las normativas higiénicas sanitarias y las indicaciones dadas por el Mintur para la etapa post Covid, de modo que se garantice la seguridad de los clientes y del personal de contacto.

\section{Conclusiones.}

- En el hotel Iberostar Grand Packard no se contabilizan los costos de no calidad. Los administrativos y el personal tienen una idea errada de lo que significan; no tienen conocimiento sobre la temática ni lo que representa para la instalación.

- No existe ningún procedimiento en el área de Regiduría de Pisos que identifique los factores que originan costos de no calidad, solo se le da tratamiento mediante compensaciones a las quejas o reclamaciones emitidas por los clientes.

- El análisis de las no conformidades existentes en el área de Regiduría de Pisos permitió identificar como factores que originan costo de no calidad de fallas internas: ausentismo del personal, reprocesos del servicio, ineficiente capacitación de los trabajadores y gasto de energía eléctrica.

- Los factores que originan fallas externas en el área de Regiduría de Pisos son: el servicio de lavandería, la reposición de amenidades, el avituallamiento, decoración y limpieza de las habitaciones, siendo este último el que más insatisfacción origina a los huéspedes, representando aproximadamente el $50 \%$ de las quejas reportadas.

- Se contabilizaron los costos de no calidad originados en el área en el año 2019 por compensación a los clientes y pérdida de estos, evidenciándose una afectación en los ingresos del hotel de \$2 129.00 MLC y \$ 85050.00 MLC respectivamente.

- Se propusieron a partir de los resultados obtenidos 13 acciones de mejoras, con el fin de atenuar las deficiencias identificadas.

\section{Referencias bibliográficas.}

American Society for Quality. ASQ. (2018). Speaking of Quality. USA. Recuperado de: www.qualitymag.com. (Fecha de Acceso: 30/04/20).

Asociación Española de Contabilidad y Administración. AECA. (1995). Costos de Calidad. Documento sobre principios de contabilidad de Gestión 11. Madrid. España. 
Campanella Jack (1997). Fundamentos de los costos de la calidad. Mc Graw Hill. D.F. México.

Casermeiro, M. Scheuber, Y. y Varas, D. (2010). Algunas nociones sobre los costos de no calidad. Oficina de Calidad de los Servicios. Secretaría General de la Gobernación de Salta. España.

Colunga, C. y Saldierna, A. (1994). Los costos de calidad. Panorama. D.F. México.

Crosby Philip. (1997). La calidad no cuesta, el arte de cerciorarse de la calidad. Continental. D.F. México.

Cuatrecasas, Ll. Carrión, D. Carvajal, J. Krovina, S. Zumárraga, F. (2010). Gestión integral de la calidad. Implementación control y certificación. Profit Editorial. Recuperado de: www.qconsultores. com . (Fecha de acceso: 04/03/20).

Del Toro, M. (2011). La limpieza y el confort higiénico en los hoteles. Revista electrónica de la Agencia de Medio Ambiente. (21). Matanzas. Cuba.

Domínguez, M. (2019). Gestión por procesos en el área de alojamiento hotelero. Cuba. Recuperado de https:/www.gestiopolis.com/gestion-por-proceso-en-el-areadealojamiento-hotelero-cuba/. (Fecha de acceso: 25/02/20).

Fernández, R. y Escalona, M. (2014). El control de costos en la restauración. Editorial Félix Varela. La Habana. Cuba.

Flores, J. y Humberto, R. (2015). La administración de costos: factor de competitividad en las pequeñas y medianas empresas. X Asamblea General de la Asociación Latinoamericana de Facultades y Escuelas de Contaduría y Administración. Universidad Colima. México. Recuperado de: https://www.researchgate.net/publication/242560696. (Fecha de acceso: 19/02/20).

Gallego, J. (2007). Gestión de Hoteles: Una Nueva Visión. Editorial Thompson. Paraninfo. Madrid. España.

González Arias, M; Frías-Jiménez, R.A. y Gómez-Figueroa, O. (2014). ROTUR, Revista de Ocio y Turismo, (7). Recuperado de: http://www.rotur.es . (Fecha de acceso: 04/03/20).

Hernández, R. Baptista, P. y Fernández, C. (2010). Metodología de la Investigación. Cuarta edición. McGraw Hill. Interamericana Editores. S.A.

Lattin, G. (2012). Administración moderna de hoteles y moteles. Editorial: Trillas. D.F. México.

Mahal, I y Hossain, A. (2015). Activity-Based Costing (ABC) - An Effective Tool for Better Management. Research Journal of Finance and Accounting, 6(4)

Meneses, Y. y Rodríguez, Y. (2010). Clasificación y Planificación de los costos de calidad en el Órgano Superior de Dirección Empresarial de la Unión Eléctrica. Revista de la 
Facultad de Contabilidad y Finanzas de la Universidad de la Habana. La Habana. Cuba.

Morillo, M. y Casas, S. (2017). Sistema de costos basado en actividades en hoteles cuatro estrellas del estado Mérida, Venezuela INNOVAR. Revista de Ciencias Administrativas y Sociales, 27 (64). Universidad Nacional de Colombia Bogotá. Colombia.

Prado-Chaviano, E. Cruz-Suárez, Y. Baujín-Pérez, P. y Abreu, P. (2016). Procedimiento para la determinación de los costos de calidad. Caso de estudio hotelero. Universidad de Matanzas. Matanzas. Cuba.

Pyzdek Thomas (1996). The complete guide to the CQM. Quality Pubishing, Tucson.

Sarmiento, L. (2010). Colección de Manuales de Gestión de Calidad-Introducción a los Costos de Calidad. Segunda Edición. Recuperado de: http:// www.iso9000plus.com.ar (Fecha de acceso: 23/02/20).

Shrouty, V y Tiwari, P. (2017). Analysis of Cost of Poor Quality and its Calculation: Steel Industry. International Research Journal of Engineering and Technology (IRJET), $5(4)$.

Sower, V. y Broussard, E. (2018). Cost of quality usage and its relationship to quality system maturity International Journal of Quality \& Reliability Management 2(24).

Thomasson, M. y Wallin, J. (2013). Cost of Poor Quality; definition and development of a process-based framework. Master of Scienc Thesis in the Master Degree Programme Quality and Operations Management. Chalmers University of Technology. Suecia.

Ulacia, Z. (2016). Gestión de Alojamientos. Texto Básico de la asignatura Gestión de Alojamiento. Universidad de la Habana. Facultad de Turismo. Cuba.

Yera, A. Tellería, A. Hernández, W. Gómez, L. Nieblas, M. (2015). Cálculo de costos de calidad en la villa turística Yaguanabo de la cadena Islazul de Cienfuegos Revista Eumednet.

\section{Ciencia}




\section{Para citar el artículo indexado}

Matos Murcia, D. A. (2020). Diagnóstico de los costos de no calidad en el área de Regiduría de Pisos del hotel Iberostar Grand Packard. AlfaPublicaciones, 3(1), 33-50. https://doi.org/10.33262/ap.v3i1.38

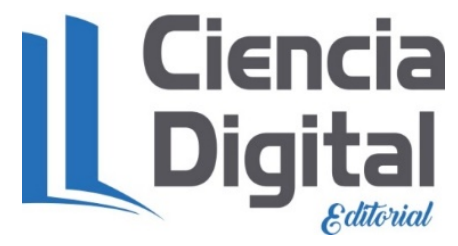

El artículo que se publica es de exclusiva responsabilidad de los autores y no necesariamente reflejan el pensamiento de la Revista Alpha Publicaciones.

El artículo queda en propiedad de la revista y, por tanto, su publicación parcial y/o total en otro medio tiene que ser autorizado por el director de la Revista Alpha Publicaciones.
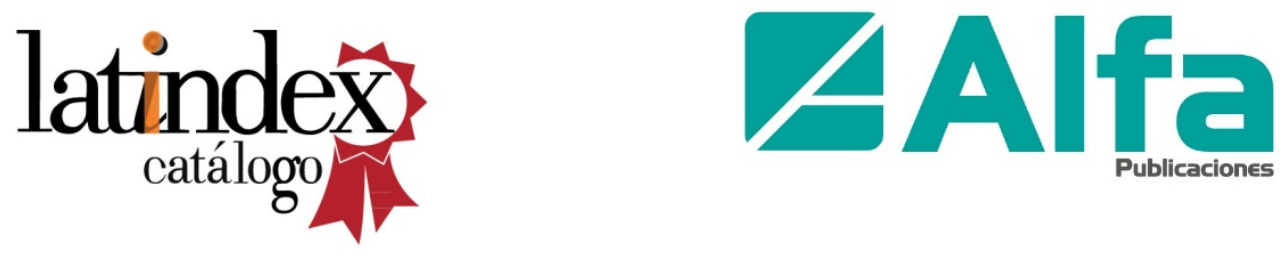